\title{
Retrospective study assessing the learning curve and the accuracy of minimally invasive robot- assisted pedicle screw placement during the first 41 robot-assisted spinal fusion surgeries
}

\author{
Joseph Maalouly ${ }^{1}$, Mehul Sarkar ${ }^{2}$, John Choi $^{1}$ \\ 'Department of Orthopedic and Spine Surgery, Peninsula Private Hospital, Frankston, VIC 3199, Australia. \\ ${ }^{2}$ Department of Orthopedic and Spine Surgery, Kaslival Hospital, Nashik, Maharashtra 422001, India.
}

Correspondence to: Dr. Joseph Maalouly, Department of Orthopedic and Spine Surgery, Peninsula Private Hospital, 525

McClelland Dr, Frankston VIC 3199, Australia. E-mail: josephmaalouly2@gmail.com

How to cite this article: Maalouly J, Sarkar M, Choi J. Retrospective study assessing the learning curve and the accuracy of minimally invasive robot-assisted pedicle screw placement during the first 41 robot-assisted spinal fusion surgeries. Mini-invasive Surg 2021:5:35. https://dx.doi.org/10.20517/2574-1225.2021.57

Received: 26 Apr 2021 First Decision: 9 Jun 2021 Revised: 9 Jun 2021 Accepted: 16 Jun 2021 Available online: 17 Jun 2021

Academic Editor: Yoshihisa Kotani Copy Editor: Xi-Jun Chen Production Editor: Xi-Jun Chen

\begin{abstract}
Aim: The purpose of this study was to assess the learning curve and the accuracy of robot-assisted pedicle screw placement in the first 41 cases.

Methods: This retrospective study investigated the first 41 patients undergoing spinal fusion, whereby 250 pedicle screws were inserted with robotic assistance in a private hospital by a single surgeon. The pedicle screw accuracy was evaluated by computed tomography scan by an orthopedic surgeon according to the Gertzbein and Robbins classification. Planning time and screw placement time were noted. In addition, data about any screw malposition, a return to the operating theatre, and intraoperative repositioning were collected. The data were analyzed with Microsoft Excel.
\end{abstract}

Results: The results show a high degree of accuracy (98\%) of pedicle screw placement with a minimally invasive robot-assisted spinal fusion with no screw malposition requiring a return to the operating theatre. The learning curve improved with time, reaching a plateau at around 25 cases. 
Conclusion: This study shows a high degree of accuracy of pedicle screw placement with the robot and it shows a surgeon's improved experience with the robot with time. Further comparative studies are needed to better assess the robot's accuracy and its future in spine surgery.

Keywords: Minimally invasive surgical procedures, bone screws, vertebrae, robotics

\section{INTRODUCTION}

Pedicle screws remain the primary mode of fixation providing adequate stabilization for facilitating fusion in spinal procedures. Freehand pedicle screw insertion remains a challenging procedure owing to the many important structures near the pedicle, such as the spinal cord, nerve roots, and associated vessels. Complications such as neurological deficits and vascular injuries secondary to misplaced pedicle screws are prevalent with an incidence of $1 \%-54 \%{ }^{[1]}$.

Moreover, complex deformities such as scoliosis and morphologic conditions such as dysplastic vertebral pedicles make screw placement challenging. The introduction of fluoroscopy has improved accuracy and facilitated the emergence of minimally invasive surgery procedures, but it has also given rise to increasing concerns of radiation exposure to the surgical team ${ }^{[2]}$. Recent availability and acceptance of computerassisted navigation techniques have minimized the risk of radiation exposure to the surgical team and improved the accuracy of screw placement, but this is largely dependent on the surgeon's expertise and knowledge of anatomy ${ }^{[3]}$.

The navigation-assisted spinal robotic system (ExcelsiusGPS ${ }^{\bullet}$, Globus Medical, Inc., Audubon, PA) used in this study is a floor-mounted guidance arm system that allows for placement of pedicle screws along the preplanned trajectory with real-time navigation guidance. Initial results show increasing accuracy of screw placement in prone and lateral position surgeries, allowing for reduction in surgical time for anterior interbody fusion with posterior instrumentation and overall radiation exposure to the surgical team ${ }^{[4]}$. However, the learning curve of this new technique needs further evaluation.

We aimed to evaluate the learning curve and describe our experience of using the ExcelsiusGPS robot in the setting of prone and lateral position surgeries, emphasizing the surgeon's experience with planning, accuracy of implant insertion, and radiation exposure incurred in our cases.

\section{METHODS}

\section{Patient selection}

The first 43 consecutive patients were evaluated, operated by a single surgeon experienced in the use of stereotactic navigation, using the ExcelsiusGPS ${ }^{\circledR}$ (Globus Medical, Inc., Audubon, PA) at a single institution from April 2019 to February 2020. All consenting patients more than 18 years of age were included irrespective of any antecedent surgical procedures. Written informed consent was obtained from all patients. All data were obtained in the outpatient clinic with follow up period of at least one year. Two cases were removed from the study cohort due to technical malfunction in the robot. The robot was successfully used in the other 41 cases. The patient demographic data (age, sex, and BMI) were recorded. Patient position, image acquisition protocol, total time of robot use, planning time for each case, and radiation exposure were noted immediately after the procedure. Two patients underwent scoliosis deformity correction. The rest were one-, two-, or three-level interbody fusions. 
Biplanar fluoroscopy was used to check the position of the screws after placement and any unacceptable screws were revised immediately and noted. Postoperatively erect radiographs were taken to check on the construct. Follow up was planned using our standard protocol with X-ray radiographs at one, three, and twelve months postoperatively and computed tomography (CT) cans at six months postoperatively of instrumented levels for all patients.

\section{Analysis}

The accuracy of pedicle screw placement was determined as grades (Grade A, $0 \mathrm{~mm}$; Grade B, 0-2 mm; Grade C, 2-4 mm; Grade D, 4-6 mm; and Grade E, > $6 \mathrm{~mm}$ ) based on the $2 \mathrm{~mm}$ incremental system according to CT scans at six months developed by Gertzbein and Robbins ${ }^{[5]}$. The number of screw breaches was recorded for each group. All revised screws were considered as inaccurate. All other screws were considered for analysis irrespective of intraoperative change of trajectory or freehand placement of screws using the ExcelsiusGPS navigation system. The number of acceptable screws divided by the number of total screws placed with robotic navigation resulted in an accuracy percentage for this study.

\section{Surgical technique}

Patients were positioned prone or lateral. The position was determined based on the surgeon's preference and the surgical procedure planned. The patients who underwent posterior only instrumentation was positioned prone on the Jackson table. The surgical field was prepped and draped in the usual sterile fashion allowing access to bilateral posterior superior iliac spine (PSIS) in all cases. The ExcelsiusGPS ${ }^{\bullet}$ fiducial marker [called the dynamic reference base (DRB)] was placed in the right PSIS via a small stab incision during prone position and in the left PSIS in the single lateral position. In cases where an open incision was utilized, the DRB was mounted on the spinous process clamp attached to the lower most spinous process not considered in the construct. The intraoperative CT fixture (ICT) was attached to the fiducial marker, in a plane parallel to the floor and just above the patient's skin over the area of interest.

Patients undergoing OLIF procedure in conjunction with posterior instrumentation were positioned in the left lateral decubitus position over a flat top table and taped in the usual fashion to allow tilting of the table without allowing any patient movement intraoperatively. The surgical field was prepped and draped widely allowing access to the left Iliac crest and PSIS. The DRB was placed on the left PSIS, while the surveillance marker was placed a few inches anterior to the DRB along the crest. The DRB post was tilted caudally to avoid any interference between the camera and the reference frame. The intraoperative CT fixture (ICT frame) was attached on the DRB post and placed flat over the area of interest on the patient's skin.

\section{Imaging protocol}

The ExcelsiusGPS robotic navigation platform supports three imaging protocols: preoperative CT scan, intraoperative CT scan, and intraoperative fluoroscopy.

\section{Preoperative CT scan}

The preoperative CT scan was utilized in patients undergoing instrumentation for more than five levels. The CT scan was carried out using a special protocol involving a scan in supine position with $1 \mathrm{~mm}$ image cuts for integration with the robotic navigation platform. In patients with preoperative CT scan, validation of landmarks was done using serial orthogonal radiographs for each level to be instrumented. The radiographs were then transferred to the robot navigation system for screw trajectory planning. The validation and verification system of the ExcelsiusGPS robot system provided alternate ghosting images of the radiographs and the CT scan during the planning phase. 


\section{Intraoperative CT protocol}

Patients undergoing less than four-level instrumentation or lateral position surgery were subjected to the intraoperative CT protocol. The O-arm-2 (Medtronic Sofamore Danek Inc, Memphis, TN) was used to acquire the three-dimensional (3D) CT scan. The surgical table was raised to a height of $110 \mathrm{~cm}$ from the floor for easy maneuvering of the O-arm doughnut without major adjustments needed for the CT spin. The surgical field was draped circumferentially in order to maintain sterility [Figure 1]. The scan was then transferred to the ExcelsiusGPS navigation system for pedicle screw trajectory planning, which included entry point, trajectory, screw length, and screw width.

During the planning phase, axial, sagittal, coronal, and $3 \mathrm{D}$ reconstruction views were available for the surgeon on the navigation screen for planning of the screw trajectories. Care was taken to use the widest and longest screws possible for each pedicle [Figure $2 \mathrm{~A}$ ].

The trajectories were matched to allow as small an incision as possible. The plan was also adjusted by the surgeon for the best possible way to allow easy rod insertion and reduction of deformity based on the surgeon's experience [Figure 2B].

Time taken for planning each screw was noted. The planning time also included sterile draping of the robot arm by the assistant done simultaneously. On validation of landmarks on the DRB with those seen on the navigation screen, the reference frame ICT was then removed from the field taking care not to disturb the DRB. The robot was then wheeled into the surgical field. The robot was docked securely to the floor once all trajectories could be reached by the end effector arm. The navigated instruments, including a position tracker, drill, and navigated screw guide, were registered in the system previously by the scrub nurse. The surgeon utilized a foot pedal to bring the robotic arm to the planned screw trajectory. With the end effector in position, a stab incision was made, and a power drill was first used to cannulate the pedicle, and the screw was then placed through the stable, rigid end effector. Real-time visualization of the screw trajectory and indicators of excessive skiving force were available to the surgeon through the process of screw insertion. Once all screws were placed, the robot was undocked and removed from the surgical field and screw placement was checked on orthogonal radiographs. Any unacceptable screws were revised immediately before advancing to the next step. Rods were inserted and the construct completed. The time taken for rod insertion was excluded from the calculation. Final A-P and lateral radiographs were taken to check the construct.

Radiation exposure: The dose imparted at each scan was calculated from the dose report generated by the $\mathrm{O}$-arm and converted to $\mathrm{mSv}$ using a uniform tissue factor of 0.015 for uniformity in calculations. Similarly, all fluoroscopy exposures were measured in milliseconds based on the readings from the C-arm.

\section{RESULTS}

The ExcelsiusGPS Spine robot guidance system was successfully used in 41 of 43 consecutive cases between April 2019 and February 2020. Two cases were abandoned due to technical reasons where the robot could not connect with the intraoperative image acquisition system. Those cases were excluded from the study. Statistical analysis was done using Microsoft Excel (Microsoft Corporation, Redmond, Washington, United States). Fixation was done across 86 motion segments over the course of the study period. The mean age of patients was $70.9 \pm 10.5$ years. Thirty $(60 \%)$ patients were female and $20(40 \%)$ were male [Table 1 ]. The mean BMI was 29.2. Of the 41 patients, 17 patients were operated in the single lateral position; 8 patients were operated in the lateral position and then repositioned in prone position for posterior fixation; and 16 patients were operated in prone position only. Out of the 41 lumbar fusion patients, 33 had interbody fusion 
Table 1. Demographics

\begin{tabular}{ll}
\hline Age (years) & $70.9 \pm 9.5$ \\
M/F & $15 / 26$ \\
Total cases $(n)$ & 41 \\
BMI & $29.2 \pm 5.6$ \\
Total screws for assessment & 250 \\
Total number of instrumented levels & 86 \\
\hline
\end{tabular}

BMI: Body mass index.

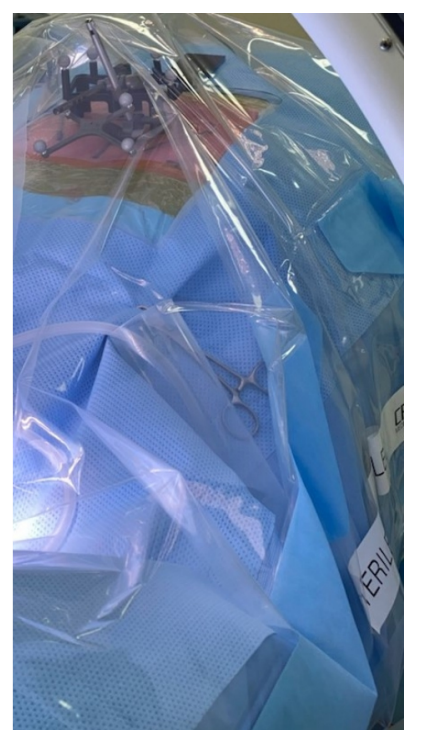

Figure 1. Draping of the patient when O-arm is used with DRB and ICT shown clearly. DRB: Dynamic reference base; ICT: intraoperative computed tomography.
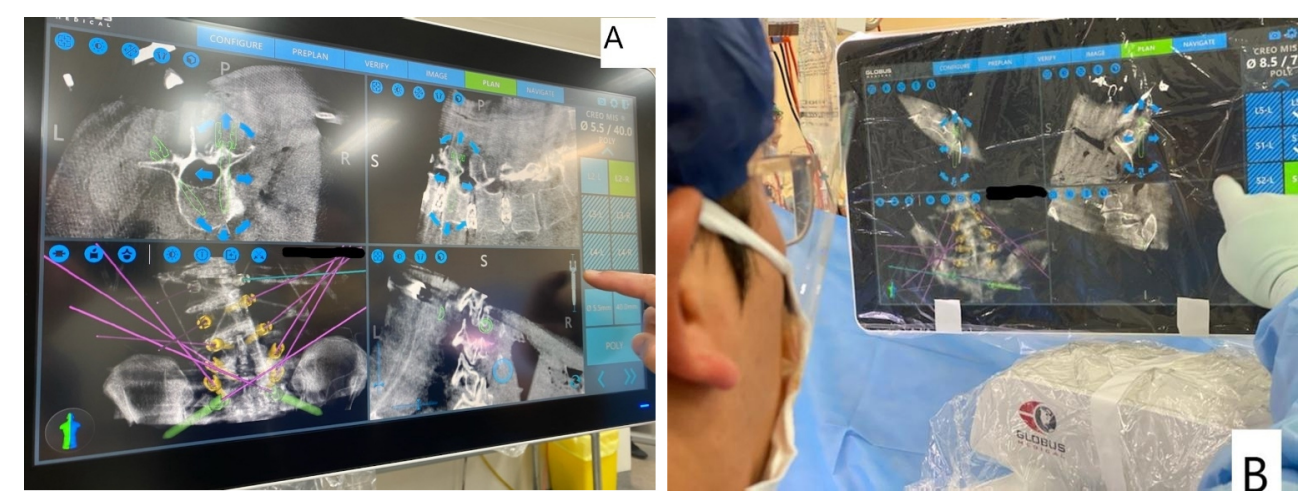

Figure 2. (A) Intraoperative planning prior to draping. (B) Intraoperative planning change with the screen draped during screw insertion.

and reconstruction. The intraoperative CT scan protocol was utilized in 38 cases. The preoperative CT scan with intraoperative fluoroscopy integration was used in three patients.

\section{Learning curve}

The patients were classified into five groups. Group A, Cases 1-9; Group B, Cases 10-18; Group C, Cases 1926; Group D, Cases 27-32; and Group E, Cases 33-41 [Table 2]. The surgeon's learning curve was reached at 
Table 2. Group characteristics of the study

\begin{tabular}{llllll}
\hline Variable & Group A (9) & Group B (9) & Group C (8) & Group D (6) & Group E (9) \\
\hline Screws inserted & 56 & 60 & 46 & 38 & 50 \\
Screws revised & 0 & 1 & 1 & 0 & 3 \\
Intraoperative trajectory revision & 0 & 1 & 2 & 1 & 1 \\
Unreachable trajectory & 6 & 2 & 0 & 0 & 0 \\
Grade A + B Screws & 54 & 58 & 42 & 29 & 43 \\
Grade C screws & 2 & 2 & 0 & 0 & 7 \\
Grade D, E screws & 0 & 0 & & & 0 \\
\hline
\end{tabular}

around Group C. In Group A, the robot could not reach all the planned screw trajectories requiring it to be repositioned in four cases. The reason was a steep sacral slope in three of these patients which was not accounted for while planning the trajectory of the screw, causing the robot arm to press on the rib cage, which obstructed achieving the final trajectory. In one other patient, the robot arm was obstructed by the operating table frame in the lateral position surgery. The unachieved trajectories were cannulated by hand using the navigation function of the robot system. No repositioning was needed in the following case groups as understanding and experience improved the trajectory planning. The surgeon showed a serial decrease in time taken to plan at each level, with an average of $4.1 \mathrm{~min}$ of planning per level. As this was part of the learning curve of the surgeon with this new technology, all planning and screw placement were done by the surgeon. Cases in Group E reported a longer planning time of 5.08 min per level due to educating and teaching of visiting surgeons. The two outlier cases where teaching occurred were omitted, and the scatterplot obtained showed a decrease in planning time [Figure 3].

In total, 250 screws were inserted using the robotic arm guidance to fix 86 motion segments. Fourteen screws were inserted without the use of the robot arm. The robot failed to reach its trajectory in eight screws, and they were inserted using the navigation system of the ExcelsiusGPS robot. Five screws required an intraoperative adjustment of trajectory. Five screws had to be revised. The revised screws were reported as one in Group B, on in Group C, and three in Group E. No conclusion could be derived from the increasing frequency of revised screws in the last group. However, the average insertion time of screws using the robot showed a decreasing trend [Figure 4].

The mean radiation characteristics of our experience are summarized in Table 3. The mean fluoroscopy dose in seconds per case was around $16.32 \pm 13.22 \mathrm{~s}$ with the mean effective dose of $8.2 \pm 3.74 \mathrm{mSv}$ during the $3 \mathrm{D}$ CT scan. One patient needed a repeat scan as the CT scan was inverted and could not be validated by the robot. Fluoroscopy exposure was high in cases that required manual insertion, causing multiple shots to evaluate screw positioning. Moreover, a high radiation dose was recorded in the three cases where the preoperative CT protocol was used due to difficulty of fluoroscopy integration to match the CT images to the patient's anatomy.

\section{Accuracy}

In total, 250 screws were analyzed for accuracy on CT scan. S2 alar iliac screws as part of lumbosacral construct were deemed acceptable if they did not breach the sacral foramen or breach the pelvic wall. The overall accuracy in this study was 98\%. Overall, 245 screws were deemed acceptable with 226/250 (90.4\%) being Grade A + B and 24/250 (9.6\%) being Grade C [Figure 5].

Five screws were revised immediately after confirming on fluoroscopy and before rod placements. Those were considered inaccurate. The inability of the robot to reach screw trajectory was high in Group A cases 
Table 3. Radiation exposure recorded from the study

\begin{tabular}{ll}
\hline Mean effective dose from O-arm spin & $8.2 \pm 3.74 \mathrm{mSv}$ \\
Mean fluoroscopy dose & $16.32 \pm 13.22 \mathrm{~s}$ \\
Mean fluoro time in preoperative CT protocol (for integration) & $54 \mathrm{~s}$ \\
\hline
\end{tabular}

CT: Computed tomography.

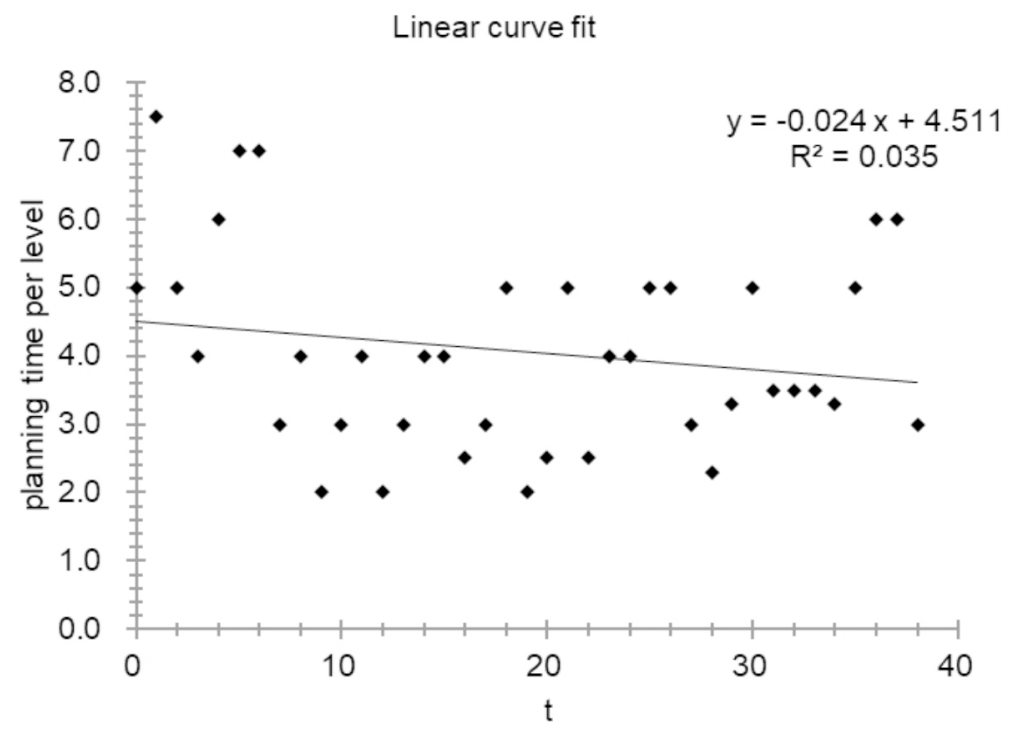

Figure 3. Scatterplot of planning time per level learning curve. The $x$-axis shows the case number as a function of planning time per level (results are based on 39 patients with the two outliers due to teaching purposes eliminated).

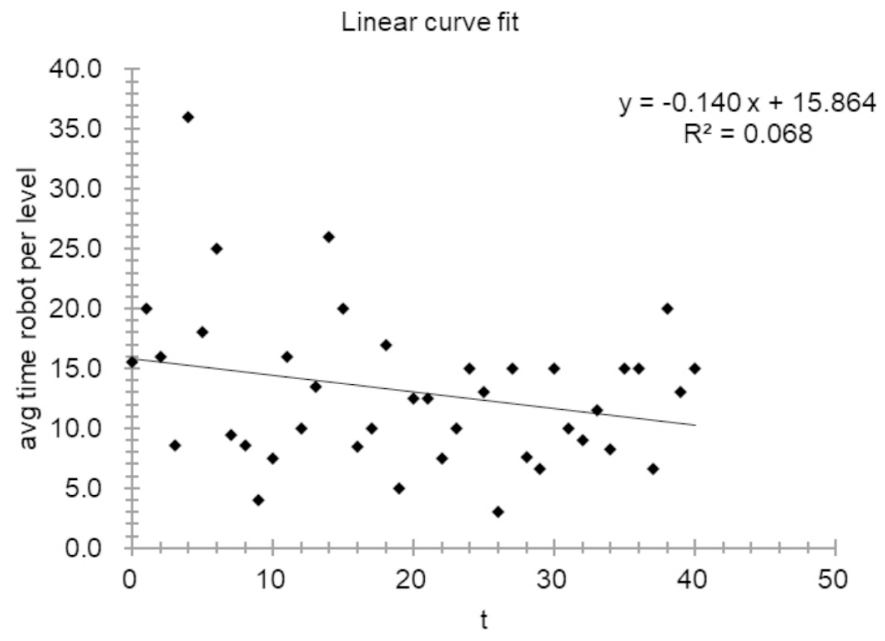

Figure 4. Scatterplot of average screw insertion time with robot as a function of cases.

$(6 / 56)$ and declined in the subsequent cases. However, the last group had a rise in screw misplacements which were revised intraoperatively (3/50). This was probably due to skipping a step of validation of ICT in one instance, movement of the surveillance marker in the second instance, and error in planning in the third instance. None of the patients returned to the theatre to revise a screw. 

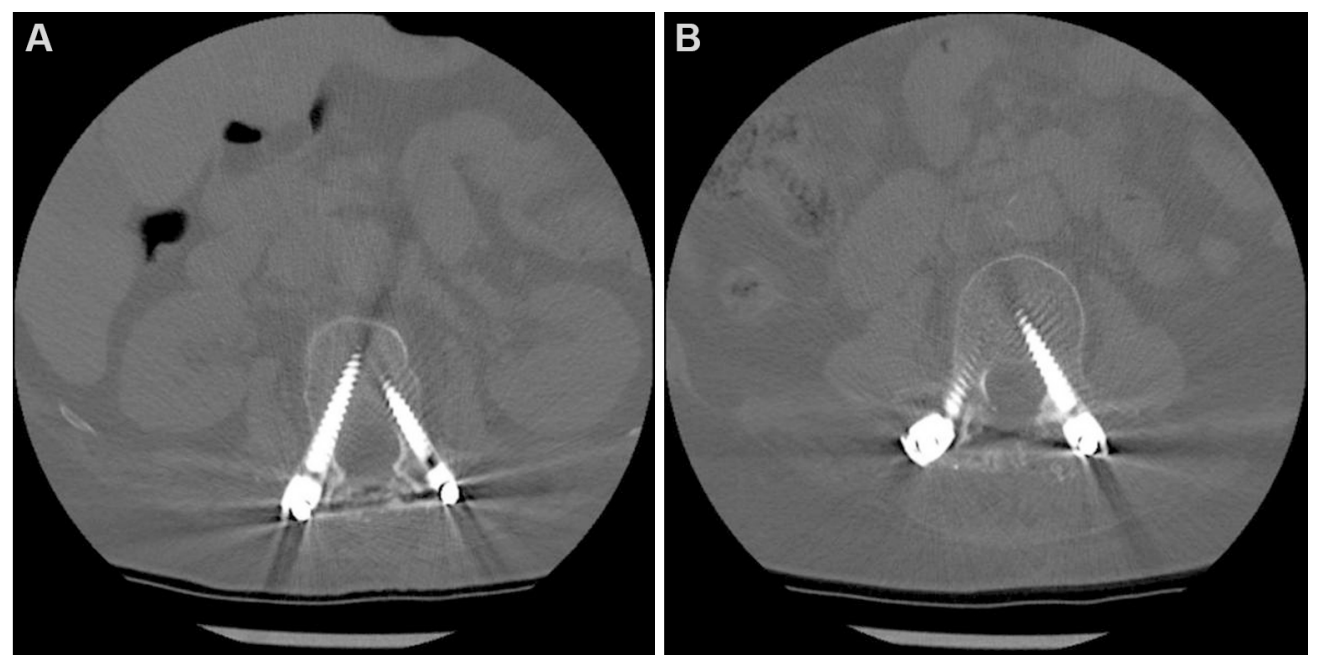

Figure 5. Axial CT scan showing: (A) Grade A on the left pedicle screw; and (B) Grade B on the right pedicle screw. CT: Computed tomography.

\section{DISCUSSION}

Robotics in spinal surgery has been developing rapidly over the last decade, designed to augment and enhance the surgeon's abilities. Freehand placement of pedicle screws requires detailed knowledge of anatomical landmarks and surgeon experience. The use of imaging technology such as fluoroscopy and intraoperative navigation has improved the safety and accuracy of pedicle screw placement over freehand techniques ${ }^{[3]}$. The meta-analysis of 30 studies analyzing 9000 pedicle screws by Mason et al. ${ }^{\left[{ }^{[6]}\right.}$ concluded that traditional fluoroscopy reached an accuracy of $63.1 \%$, two-dimensional navigation had $84.3 \%$ accuracy, and 3D navigation was most accurate at $95.5 \%^{[7]}$. Many new robotic spine guidance systems are being developed, improving the safety and accuracy of pedicle screw placement. However, the learning curve of these systems has not been studied in detail. There was insufficient evidence to conclude the effectiveness of robot-assisted over conventional fluoroscopy-guided pedicle screw insertion in a systematic review by Marcus et al. ${ }^{[8]}$. On the contrary, another study reported equivalent accuracy with reduced radiation exposure in robot-assisted cases compared to conventional fluoroscopy-guided surgery ${ }^{[\rho]}$.

Multiple studies have reported on the accuracy of robot-assisted pedicle screws using a variety of classification systems. In multiple systematic meta-analyses, it was concluded that the $2 \mathrm{~mm}$ incremental classification system developed by Gertzbain and Robbins has been widely accepted and used for the assessment of pedicle screw placement accuracy on CT scans ${ }^{[10,11,12]}$. Theologou et al ${ }^{[13]}$ reported good interobserver reliability and commented on the ease of using this system. Hu et al. ${ }^{[14]}$ reported a high accuracy of 98.9\% using the Renaissance system but used postoperative radiographs for the assessment which was a major limitation of the study. Similarly, Pechlivanis et al. ${ }^{[15]}$ used the Renaissance system and reported that 91.5\%-98.3\% of screws were placed in an acceptable position as per the Gertzbein and Robbins classification system. Keric et al. ${ }^{[16]}$ also reported a higher accuracy of $96.7 \%$ in the placement of 2067 screws using the assessment system described by Wiesner et al. ${ }^{[17]}$. In this study, the overall accuracy was $98 \%$ using the $2 \mathrm{~mm}$ incremental system of CT scan. Out of 250 screws placed using the robot, five screws (2\%) were revised due to unsatisfactory placement immediately after fluoroscopic confirmation. These screws were considered inaccurate. According to our assessment, $90.4 \%$ of screws (Grade A + B) were placed entirely inside the pedicle and $9.6 \%$ of screws were acceptable, being Grade C. None of the screws had any major pedicle breach or associated clinical symptoms. This was probably because the surgeon could modify the planned trajectory if there was excessive force or skiving of the drill causing malposition of screws. This helped 
improve the accuracy of screw placement. Huntsman et al. ${ }^{[4]}$ also reported a similar high accuracy of $99 \%$ using the ExcelsiusGPS system in a study of 100 cases.

The single position lateral surgery has not gained widespread approval due to technical difficulty of cannulating a pedicle screw in the lateral position. Recent studies have reported a reduction in operative time in single position lateral pedicle screw fixation following lateral interbody fusion as compared to dual positioning without an increase in complication rates or compromised perioperative outcomes ${ }^{[18-20]}$. The authors believe the use of the rigid robot arm of the ExcelsiusGPS system will provide a stable and accurate insertion of pedicle screws in the lateral decubitus position. The accuracy of screw placement in the subset of patients operated in a single lateral position was $97.87 \%$, where two screws were revised out of 94 pedicle screws. A similar high accuracy of $98 \%$ was reported by Huntsman et al. ${ }^{[4]}$ using the ExcelsiusGPS robot system. Surgeons operating in a single lateral position surgery face the technical challenge of cannulating pedicle screws in this position and some technique-related complications; however, recent studies showed comparable results to the flip position ${ }^{[18-20]}$. Furthermore, as the placement of S2AI screws can be challenging by the freehand technique, the robot can be used to place these screws ${ }^{[2,22]}$.

The learning curve for accurate planning and execution of screw placement was reached in Group C in this study. The time taken to plan screw trajectories saw a significant reduction in the initial period of this study. The mean time taken to plan in Group A was $5.35 \mathrm{~min}$ and in Group B was $3.15 \mathrm{~min}$. The robot arm could not reach planned trajectories in six instances in Group A and once in Group B. The authors believe this reduction in missed trajectories was due to improved planning and increasing familiarity of the $3 \mathrm{D}$ interface. However, the planning time increased in the following case groups as the planning interface was utilized as an academic tool to train fellows and visiting surgeons.

Similarly, this study demonstrates a gradual reduction in screw insertion time which is comparable to other studies. Urakov et al. ${ }^{[23]}$ studied the mean insertion times for trainee surgeons using the older generation robotic guidance system and reported a mean time of 5.7 min per screw for percutaneous screw placement and 3.6 min per screw for freehand insertion. In our study, the initial case (Groups A and B) showed a declining trend in screw insertion time from $5.23 \mathrm{~min}$ per screw to $4.36 \mathrm{~min}$ per screw. The least amount of average time per screw was reached around the Group C mark with a mean time of 3.78 min per screw.

Radiation exposure in image-guided surgery is a significant concern to the surgical team. The use of robotic-assisted navigation has been postulated to reduce radiation exposure as compared to traditional fluoroscopy-based surgery. A recent study reported significantly lower radiation times in the robot-assisted group than in the fluoroscopy-guided group ${ }^{[24]}$. However, the cumulative radiation exposure reported during surgery in the robot-assisted group was $93.5 \pm 37.9 \mathrm{~s}$, which was significantly higher than what was found in our study $(16.32 \pm 13.22 \mathrm{~s})$. Similarly, Pennington et al ${ }^{[25]}$ conducted a systematic review which found that robot-assisted surgery with preoperative CT imaging had significantly less radiation exposure than in fluoroscopy-based surgery. The researchers estimated a dose of 14-16 mSv in fluoroscopy-navigated surgeries as compared to $4.8 \mathrm{mSv}$ exposure dose of intraoperative O-arm scan for CT navigation-based surgeries. This estimation, although less than the one encountered in this study ( $8.2 \pm 3.74 \mathrm{mSv}$ ), can be explained by the need for a high-dose wide-view CT scan for validation of the ExcelsiusGPS system. Even though the CT radiation dose in this study was higher than that of the $\mathrm{O}$-arm scan utilized in navigationonly systems (Costa et al. ${ }^{[26]}$ ), it is significantly lower than that encountered in a fluoroscopy-based procedure. The mean fluoroscopy time reported by these researchers was $20.1 \pm 17.2 \mathrm{~s}$ per screw, which was significantly higher than the one reported in this study (16.32 $\pm 13.22 \mathrm{~s}$ per case). Benech et al. ${ }^{[2]}$, in a study on the initial experience and radiation exposure using a similar robotic guidance system, reported a 
fluoroscopy time of $17.6 \pm 17.4 \mathrm{~s}$ for the entire case series, which is significantly less compared to our finding of $54 \mathrm{~s}$ in the group of cases operated using the preoperative CT protocol. The authors believe the complex deformity reconstruction and instrumentation at more than five levels was the reason for the higher fluoroscopy exposure in this study, as it took more than two orthogonal radiographs per level for accurate validation of anatomical landmarks for integration of the preoperative CT scan.

The use and acceptance of new technology always raises important questions regarding the development of skill and the ease of introduction of the new technique in clinical practice. Robot-assisted spine surgery faces the same challenges. All new technologies and techniques have a learning curve that a surgeon must overcome to be proficient in its use. In the authors' opinion, the learning curve for this technique lies around the 25 cases mark. The authors believe supervised training in the initial period can help reduce the learning curve for improved surgical outcomes. This study showed acceptable accuracy in the placement of pedicle screws, but, ultimately, it depends on the meticulous planning and effective execution of the plan.

\section{Limitations}

There are several limitations to this study. Even though this is a single-center study, the patients were not randomized and lacked a comparative control. Even though our study reports excellent accuracy in the placement of pedicle screws with reduced radiation exposure, robotic navigation systems are not common due to the large financial commitments involved. A larger sample size is needed to evaluate the overall effectiveness and cost-benefit analysis of this novel robotic navigation system.

In conclusion, robot-assisted spinal surgery is still in the initial stages of development but shows promise. Currently, the use of navigation-assisted robotics appears to provide safe and accurate placement of pedicle screws in prone as well as lateral single position surgery and appears superior to freehand screw placements, although detailed studies with larger sample size are needed for a conclusive determination. Radiation exposure encountered in this study was significantly less as compared to fluoroscopy-guided surgeries, especially in lateral access single position surgeries. We conclude that, with a short learning curve of 25 cases, robotic spine surgery seems to be useful in execution of complex deformity reconstructions. However, ultimately, it is up to the surgeon to effectively execute the planned procedure with the help of the robot system.

\section{DECLARATIONS}

\section{Authors' contributions}

Design of the work, writing and revising the manuscript, analysis and collection of data: Maalouly J

Collection of data and writing of the manuscript: Sarkar M

Design of the work, writing of the manuscript and supervision of the study: Choi J

\section{Availability of data and materials}

Not applicable.

\section{Financial support and sponsorship}

None.

\section{Conflicts of interest}

All authors declared that there are no conflicts of interest. 


\section{Ethical approval and consent to participate}

Written informed consent was obtained from all patients.

\section{Consent for publication}

A written informed consent for publication was obtained.

\section{Copyright}

(c) The Author(s) 2021.

\section{REFERENCES}

1. Molliqaj G, Schatlo B, Alaid A, et al. Accuracy of robot-guided versus freehand fluoroscopy-assisted pedicle screw insertion in thoracolumbar spinal surgery. Neurosurg Focus 2017;42:E14. DOI PubMed

2. Benech CA, Perez R, Benech F, Greeley SL, Crawford N, Ledonio C. Navigated robotic assistance results in improved screw accuracy and positive clinical outcomes: an evaluation of the first 54 cases. J Robot Surg 2020;14:431-7. DOI PubMed PMC

3. Hyun SJ, Kim KJ, Jahng TA, Kim HJ. Minimally invasive robotic versus open fluoroscopic-guided spinal instrumented fusions: a randomized controlled trial. Spine 2017;42:353-8. DOI PubMed

4. Huntsman KT, Riggleman JR, Ahrendtsen LA, Ledonio CG. Navigated robot-guided pedicle screws placed successfully in singleposition lateral lumbar interbody fusion. J Robot Surg 2020;14:643-7. DOI PubMed PMC

5. Gertzbein SD, Robbins SE. Accuracy of pedicular screw placement in vivo. Spine 1990;15:11-4. DOI PubMed

6. Mason A, Paulsen R, Babuska JM, et al. The accuracy of pedicle screw placement using intraoperative image guidance systems: a systematic review. J Neurosurg Spine 2014;20:196-203. DOI

7. Gelalis ID, Paschos NK, Pakos EE, et al. Accuracy of pedicle screw placement: a systematic review of prospective in vivo studies comparing free hand, fluoroscopy guidance and navigation techniques. Eur Spine J 2012;21:247-55. DOI PubMed PMC

8. Marcus HJ, Cundy TP, Nandi D, Yang GZ, Darzi A. Robot-assisted and fluoroscopy-guided pedicle screw placement: a systematic review. Eur Spine J 2014;23:291-7. DOI PubMed PMC

9. Gao S, Lv Z, Fang H. Robot-assisted and conventional freehand pedicle screw placement: a systematic review and meta-analysis of randomized controlled trials. Eur Spine J 2018;27:921-30. DOI PubMed

10. Aoude AA, Fortin M, Figueiredo R, Jarzem P, Ouellet J, Weber MH. Methods to determine pedicle screw placement accuracy in spine surgery: a systematic review. Eur Spine J 2015;24:990-1004. DOI PubMed

11. Tarawneh AM, Salem KM. A systematic review and meta-analysis of randomized controlled trials comparing the accuracy and clinical outcome of pedicle screw placement using robot-assisted technology and conventional freehand technique. Global Spine $J$ 2021;11:575-86. DOI PubMed PMC

12. Fatima N, Massaad E, Hadzipasic M, Shankar GM, Shin JH. Safety and accuracy of robot-assisted placement of pedicle screws compared to conventional free-hand technique: a systematic review and meta-analysis. Spine J 2021;21:181-92. DOI PubMed

13. Theologou M, Theologou T, Zevgaridis D, Skoulios N, Matejic S, Tsonidis C. Pedicle screw placement accuracy impact and comparison between grading systems. Surg Neurol Int 2017;8:131. DOI PubMed PMC

14. Hu X, Lieberman IH. What is the learning curve for robotic-assisted pedicle screw placement in spine surgery? Clin Orthop Relat Res 2014;472:1839-44. DOI PubMed PMC

15. Pechlivanis I, Kiriyanthan G, Engelhardt M, et al. Percutaneous placement of pedicle screws in the lumbar spine using a bone mounted miniature robotic system: first experiences and accuracy of screw placement. Spine 2009;34:392-8. DOI PubMed

16. Keric N, Doenitz C, Haj A, et al. Evaluation of robot-guided minimally invasive implantation of 2067 pedicle screws. Neurosurg Focus 2017;42:E11. DOI PubMed

17. Wiesner L, Kothe R, Schulitz KP, Rüther W. Clinical evaluation and computed tomography scan analysis of screw tracts after percutaneous insertion of pedicle screws in the lumbar spine. Spine (Phila Pa 1976) 2000;25:615-21. DOI

18. Xu DS, Walker CT, Godzik J, Turner JD, Smith W, Uribe JS. Minimally invasive anterior, lateral, and oblique lumbar interbody fusion: a literature review. Ann Transl Med 2018;6:104. DOI PubMed PMC

19. Ziino C, Konopka JA, Ajiboye RM, Ledesma JB, Koltsov JC, Cheng I. Single position versus lateral-then-prone positioning for lateral interbody fusion and pedicle screw fixation. J Spine Surg 2018;4:717-24. DOI PubMed PMC

20. Blizzard DJ, Thomas JA. MIS single-position lateral and oblique lateral lumbar interbody fusion and bilateral pedicle screw fixation: feasibility and perioperative results. Spine 2018;43:440-6. DOI PubMed

21. Laratta JL, Shillingford JN, Lombardi JM, et al. Accuracy of S2 alar-iliac screw placement under robotic guidance. Spine Deform 2018;6:130-6. DOI PubMed

22. Shillingford JN, Laratta JL, Park PJ, et al. Human versus Robot: a propensity-matched analysis of the accuracy of free hand: versus: robotic guidance for placement of S2 alar-iliac (S2AI) screws. Spine 2018;43:E1297-304. DOI PubMed

23. Urakov TM, Chang KH, Burks SS, Wang MY. Initial academic experience and learning curve with robotic spine instrumentation. Neurosurg Focus 2017;42:E4. DOI PubMed

24. Zhang Q, Han XG, Xu YF, et al. Robot-assisted versus fluoroscopy-guided pedicle screw placement in transforaminal lumbar interbody fusion for lumbar degenerative disease. World Neurosurg 2019;125:e429-34. DOI PubMed

25. Pennington Z, Cottrill E, Westbroek EM, et al. Evaluation of surgeon and patient radiation exposure by imaging technology in patients 
undergoing thoracolumbar fusion: systematic review of the literature. Spine J 2019;19:1397-411. DOI PubMed

26. Costa F, Dorelli G, Ortolina A, et al. Computed tomography-based image-guided system in spinal surgery: state of the art through 10 years of experience. Neurosurgery 2015;11:59-67. DOI PubMed 